\title{
Performance Analysis of Two-Dimensional Dead Reckoning Based on Vehicle Dynamic Sensors during GNSS Outages
}

\author{
Joong-hee Han, ${ }^{1}$ Chi-ho Park, ${ }^{1}$ Chang-Ki Hong, ${ }^{2}$ and Jay Hyoun Kwon ${ }^{3}$ \\ ${ }^{1}$ Intelligent Devices and Systems Research Group, Daegu Gyeongbuk Institute of Science \& Technology, 333 Techno Jungang-daero, \\ Hyeonpung-myeon, Dalseong-gun, Daegu 711-873, Republic of Korea \\ ${ }^{2}$ Department of Geoinformatics Engineering, Kyungil University, 50 Gamasil-gil, Gyeongsan-si, Gyeongbuk 712-701, Republic of Korea \\ ${ }^{3}$ Department of Geoinformatics, University of Seoul, 163 Seoulsiripdaero, Dongdaemun-gu, Seoul 130-743, Republic of Korea
}

Correspondence should be addressed to Jay Hyoun Kwon; jkwon@uos.ac.kr

Received 17 April 2017; Accepted 25 July 2017; Published 7 September 2017

Academic Editor: Lei Zhang

Copyright (C) 2017 Joong-hee Han et al. This is an open access article distributed under the Creative Commons Attribution License, which permits unrestricted use, distribution, and reproduction in any medium, provided the original work is properly cited.

\begin{abstract}
Recently, to improve safety and convenience in driving, numerous sensors are mounted on cars to operate advanced driver assistant systems. Among various sensors, vehicle dynamic sensors can measure the vehicle motions such as speed and rotational angular speed for dead reckoning, which can be applied to develop a land vehicle positioning system to overcome the weaknesses of the GNSS technique. In this paper, three land vehicle positioning algorithms that integrate GNSS with vehicle dynamic sensors including a wheel speed sensor (WSS), a yaw rate sensor (YRS), and a steering angle sensor (SAS) are implemented, and then a performance evaluation was conducted during GNSS outages. Using a loosely coupled strategy, three integration algorithms are designed, namely, GNSS/WSS, GNSS/WSS/YRS, and GNSS/WSS/YRS/SAS. The performance of the three types of integration algorithm is evaluated based on two data sets. The results indicate that both the GNSS/WSS/YRS integration and the GNSS/WSS/YRS/SAS integration could estimate the horizontal position with meter-level accuracy during 30-second GNSS outages. However, the GNSS/WSS integration would provide an unstable navigation solution during GNSS outages due to the accuracy limitation of the computed yaw rate using WSS.
\end{abstract}

\section{Introduction}

The vehicle positioning technique is a key component in car navigation to find and guide routes. Typical car navigation uses a low-cost GNSS (Global Navigation Satellite Systems) receiver that can provide the position and velocity of a vehicle with an accuracy that is appropriate for navigation. However, the major drawback of the GNSS based positioning technique is that the performance depends on the satellite signal reception environment. In particular, cars often move near buildings and tunnels where the GNSS signal environment is poor, and thus the GNSS based positioning technique cannot guarantee continuity and reliability of positioning $[1,2]$. Therefore, in order to overcome this problem, the GNSS based technique is combined with a dead reckoning (DR) sensor, which does not rely on any external sources. A well-known DR sensor is the inertial navigation system
(INS), which estimates the navigation solution by using the acceleration and angular rate measured by the inertial measurement unit (IMU) [1]. In particular, advances in MEMS (microelectromechanical system) technology have led to the development of a small-sized and low-cost INS [3], which is integrated with GNSS for a continuous and accurate solution in car navigation systems [3-5]. Although GNSS/MEMS-INS can fulfill the required performance for car navigation system, these sensors are too expensive for use in commercial navigation systems [6].

Recently, several ADAS (Advanced Driver Assistance Systems) such as ABS (Antilock Brake System), ESC (Electronic Stability Control), and ACC (Adaptive Cruise Control) have been applied to most passenger cars for driver and passenger safety [7]. Since ADAS operation relies on information about the vehicle environment and dynamics, numerous vehicle dynamic sensors and surround sensors and actuators are built 
TABLE 1: Relationship of the vehicle dynamic sensors and navigation information for DR.

\begin{tabular}{lccc}
\hline Sensor name & Velocity & Yaw rate & Others \\
\hline Wheel speed sensor (WSS) & Directly & $\begin{array}{c}\text { Derived from the differential of left and } \\
\text { right wheel speed }\end{array}$ & - \\
Steering angle sensor (SAS) & - & Derived from kinematic model & $\begin{array}{c}\text { Can estimate the side slip by kinematic } \\
\text { relationship }\end{array}$ \\
Yaw rate sensor (YRS) & & Directly & \\
\hline
\end{tabular}

in a car [8]. Among the sensors, vehicle dynamic sensors such as wheel speed sensor (WSS), steering angle sensor (SAS), and yaw rate sensor (YRS) measure and provide the vehicle motions for two-dimensional DR navigation. Therefore, as vehicle dynamic sensors can be applied to overcome the limitations of the GNSS technique, various studies combining GNSS and vehicle dynamic sensors have been conducted. Bonnifait et al. [9] developed a localization system using GPS and WSS. They showed that a two-dimensional DR navigation based on WSS could estimate the horizontal positions with meter-level accuracy with GPS. However, this study has not been fully analyzed to evaluate the performance of WSS based DR navigation during GNSS outages. Iqbal [2] and Georgy et al. [10] studied the performance of twodimensional DR navigation using the yaw rate measured by a gyro as well as velocity based on WSS in GNSS signal blockage situations. However, since these studies used the yaw rate measured from an IMU, they are limited with respect to verifying the performance of DR navigation based on vehicle dynamic sensors mounted on a car. The integration DR navigation based on a dynamic vehicle model using vehicle dynamic sensors with GNSS was studied [11, 12]. The dynamic model takes into account the vehicle dynamic characteristic such as lateral force and tire side slip angle that could estimate stable positioning results rather than the vehicle model based on the geometric relationship. However, to implement the dynamic vehicle model, the vehicle parameters such as front and rear slip angle, tire cornering stiffness, and angular speed of the wheels are required [3], and thus it is difficult to apply the above method to actual vehicle positioning.

In this study, in order to overcome the limitations of the GNSS based positioning technique, three land vehicle positioning algorithms that integrate GNSS with vehicle dynamic sensors (WSS, YRS, and SAS) are implemented, and the performance evaluation was conducted in GNSS signal blockage situations. A description of the integration strategies as well as mathematical models of three types of the GNSS/vehicle dynamic sensors is presented in Section 2. A performance evaluation of the three integration algorithms is provided in Section 3. The conclusion are given in Section 4.

\section{GNSS/Vehicle Dynamic Sensor Integration}

2.1. GNSS/Vehicle Dynamic Sensor Integration Strategies. DR is the process of estimating the current position based
TABLE 2: Description of combination strategies of vehicle dynamic sensors for DR.

\begin{tabular}{ll}
\hline $\begin{array}{l}\text { Combination } \\
\text { strategies }\end{array}$ & Description \\
\hline WSS & $\begin{array}{l}\text { (i) Nonholonomic constraints are applied in } \\
\text { lateral direction } \\
\text { (ii) WSS provides both the longitudinal } \\
\text { velocity and yaw rate }\end{array}$ \\
\hline WSS/YRS & $\begin{array}{l}\text { (i) Nonholonomic constraints are applied in } \\
\text { lateral direction } \\
\text { (ii) WSS and YRS provides the longitudinal }\end{array}$ \\
& velocity and yaw rate, respectively \\
WSS/YRS/SAS & $\begin{array}{l}\text { (i) The side slip angle is computed by SAS and } \\
\text { side } \text { (ii) YRS provides the yaw rate } \\
\text { (iii) Speed from WSS and side slip angle } \\
\text { compute longitudinal and lateral velocity }\end{array}$ \\
\hline
\end{tabular}

on the previous position using the velocity and the traveling direction measured by DR sensors. To calculate the horizontal position of the vehicle based on DR, the vehicle's velocity and yaw information are required. To apply vehicle dynamic sensors for the two-dimensional DR navigation, the relationship of the vehicle dynamic sensors and navigation information is summarized in the Table 1 .

To implement a two-dimensional DR navigation based on vehicle dynamic sensors, three different combination strategies are designed to ensure that the vehicle dynamic sensors' information is not duplicated. The strategies for twodimensional DR navigation based on vehicle dynamic sensors are summarized in Table 2.

In this study, three integration algorithms are proposed by integrating GNSS and a two-dimensional DR navigation based on combination strategies of vehicle dynamic sensors using a loosely coupled mode. In addition, three integration algorithms are implemented through the extended Kalman filter. The output of integration algorithm rate is set to $50 \mathrm{~Hz}$. The GNSS measurements are the position and the velocity calculated from the C/A code and the Doppler measurements at a GNSS receiver. The GNSS measurement update rate is set to $1 \mathrm{~Hz}$.

2.2. GNSS/WSS Integration Algorithm. A block diagram of the GNSS/WSS integration algorithm is shown in Figure 1. A two-dimensional DR based on WSS uses the speed measured at a pair of WSS of the rear wheels, and the procedure for this 


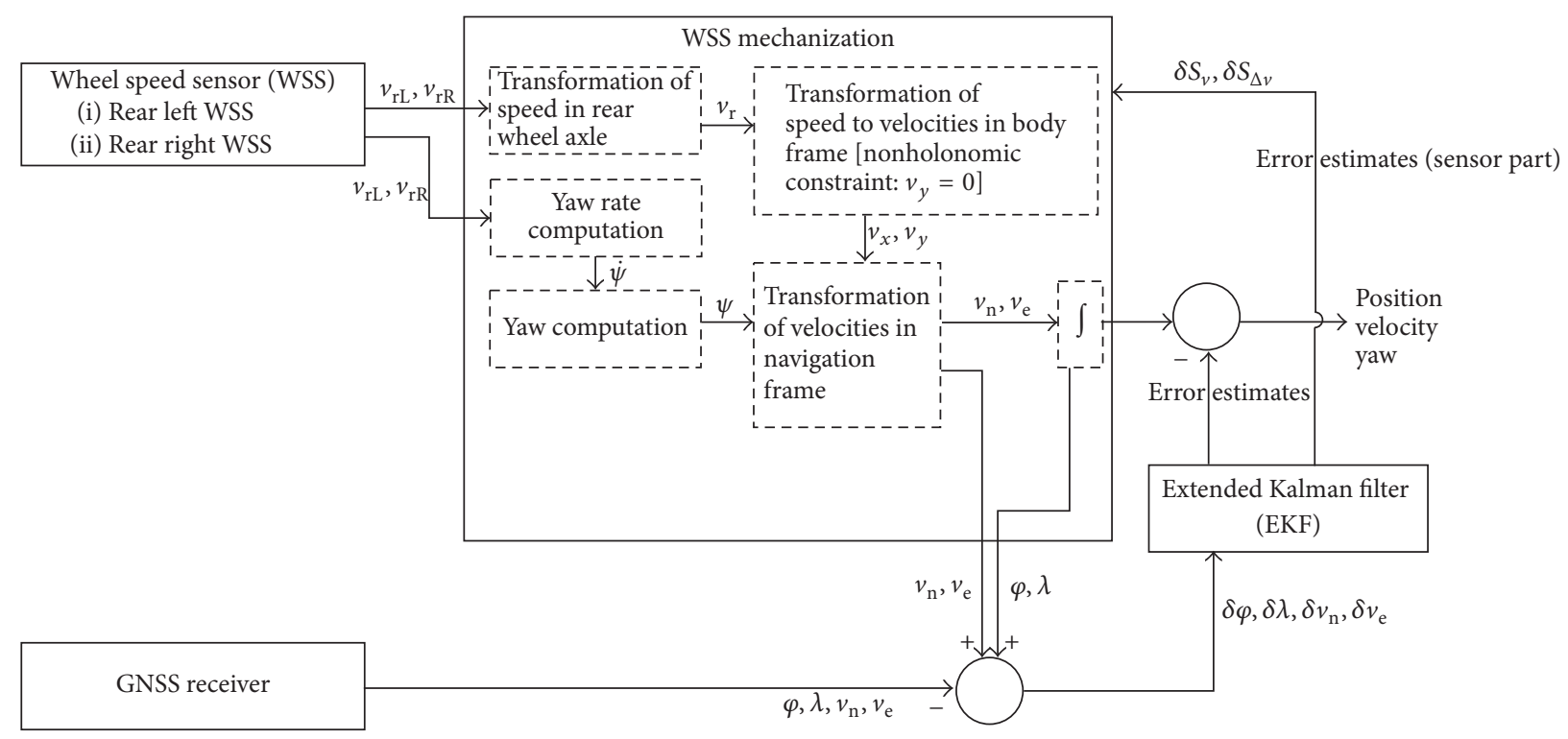

FIGURE 1: Block diagram of GNSS/WSS integration algorithm.

is as follows. When both the rear left and right WSS measure the wheel speed, the vehicle's speed, $v_{\mathrm{r}}$, is calculated at the center of the rear wheel axle as follows [13]:

$$
v_{\mathrm{r}}=\frac{v_{\mathrm{rL}}+v_{\mathrm{rR}}}{2},
$$

where $v_{\mathrm{rL}}$ and $v_{\mathrm{rR}}$ are the rear left and rear right WSS measurements, respectively.

To define the velocity in the body frame using the vehicle's speed, we assume that the center of the body frame is set to the center of the rear wheel axle, the vehicle drives on a flat road, and no wheel slip occurs. In addition, assuming that the direction of the vehicle's speed at the rear axle is equal to the longitudinal axis of the body frame, the velocities in the body frame are defined as follows:

$$
\left[\begin{array}{l}
v_{x} \\
v_{y}
\end{array}\right]=\left[\begin{array}{l}
v_{\mathrm{r}} \\
0
\end{array}\right],
$$

where $v_{x}$ and $v_{y}$ are the longitudinal and lateral velocity in the body frame, respectively.

When a vehicle turns, the left and right wheel speeds are different. The speed measured by the individual WSS varies from along track while the vehicle is turning [1]. Therefore, the yaw rate could be calculated as the difference between the rear left wheel speed and the rear right wheel speed divided by the length between the rear wheels [13], as expressed in

$$
\dot{\psi}=\frac{v_{\mathrm{rL}}-v_{\mathrm{rR}}}{T_{\mathrm{r}}},
$$

where $\dot{\psi}$ is the yaw rate of vehicle and $T_{\mathrm{r}}$ is the distance between the rear wheels.

The yaw angle of the vehicle could be computed by numerical integration using the yaw at the previous time and the yaw rate, which is written as follows:

$$
\psi(t)=\psi(t-1)+\dot{\psi} \Delta t
$$

where $\psi(t)$ and $\psi(t-1)$ denote the yaw at time $t$ and $t-1$, respectively, and $\Delta t$ is the sampling time interval.

The velocities in the navigation frame could be transformed by using

$$
\left[\begin{array}{c}
v_{\mathrm{n}} \\
v_{\mathrm{e}}
\end{array}\right]=\left[\begin{array}{cc}
\cos \psi & -\sin \psi \\
\sin \psi & \cos \psi
\end{array}\right]\left[\begin{array}{l}
v_{x} \\
v_{y}
\end{array}\right],
$$

where $v_{\mathrm{n}}$ and $v_{\mathrm{e}}$ denote the north velocity and the east velocity in the navigation frame, respectively, $\psi$ is the yaw angle at the current time, and $v_{x}$ and $v_{y}$ are the longitudinal and lateral velocity in the body frame, respectively.

In EKF-based GNSS/WSS integration, the state vector of the navigation error is composed of the latitude and longitude error, the north and east velocity error, and the yaw error. The state vector of the sensor error is composed of the average and the difference of the left and right WSS scale factor, defined as random constants. The white noise vector includes the white noise of WSS derived vehicle speed and the white noise of WSS derived yaw rate. The dynamic model for the GNSS/WSS integration is given in 


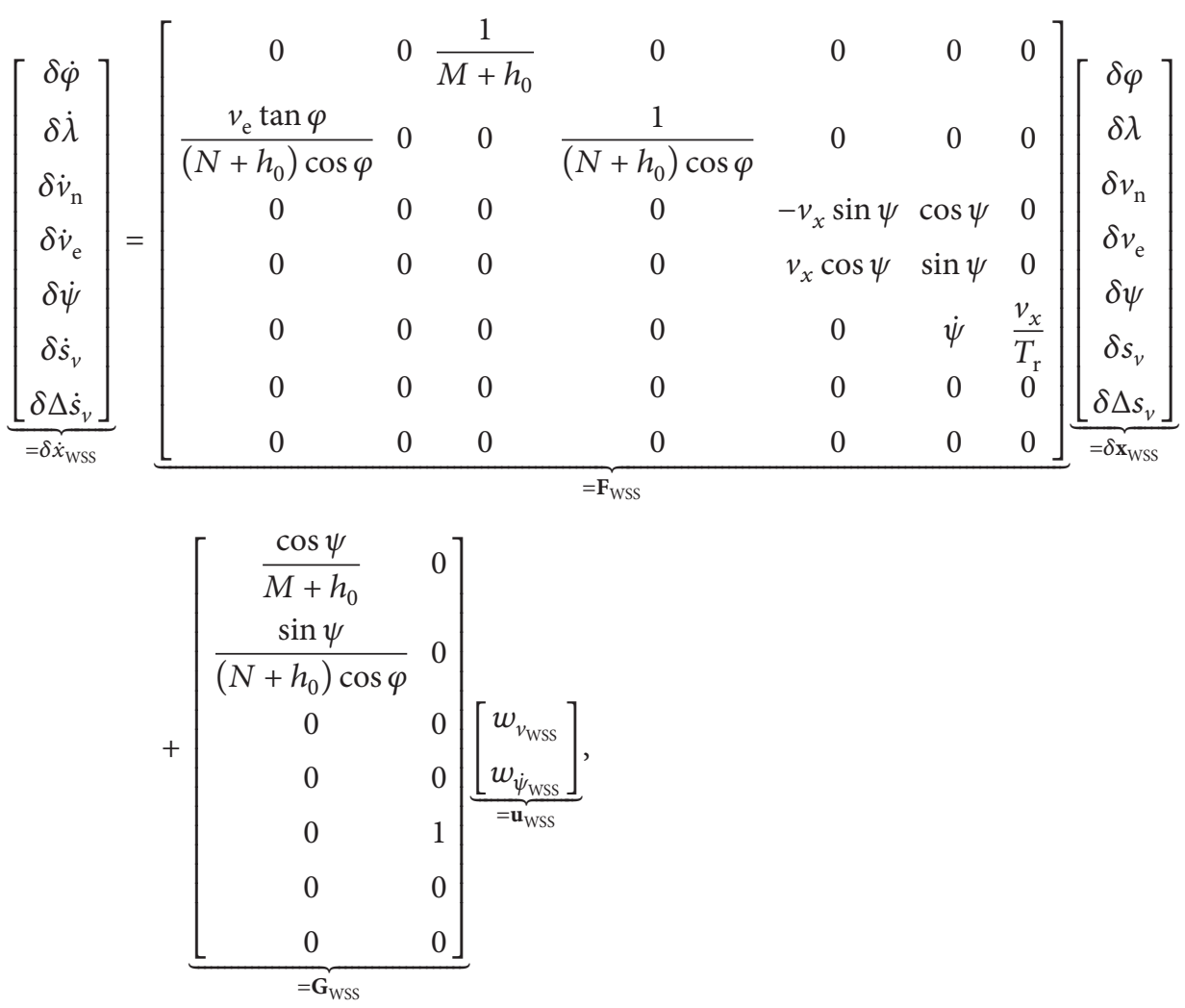

where $\mathbf{F}_{\mathrm{WSS}}, \delta \mathbf{x}_{\mathrm{WSS}}, \mathbf{G}_{\mathrm{WSS}}$, and $\mathbf{u}_{\mathrm{WSS}}$ are the dynamic matrix, the error state vector, the shaping matrix, and the white noise vector for GNSS/WSS integration, respectively, $\delta \varphi$ and $\delta \lambda$ are the latitude and longitude errors, respectively, $\delta v_{\mathrm{n}}$ and $\delta v_{\mathrm{e}}$ are the north and east velocity errors, respectively, $\delta \psi$ is the yaw error, $\delta s_{v}$ and $\delta \Delta s_{v}$ are the average and the difference of the left and right WSS scale factors, respectively, $M$ is the radius of curvature of the meridian, $N$ is the radius of curvature of the prime vertical, $h_{0}$ is the ellipsoidal height defined as a constant, $v_{x}$ and $v_{\mathrm{e}}$ are the longitudinal and the east velocity, respectively, $\varphi$ is the latitude, $\psi$ is the yaw, $T_{\mathrm{r}}$ is the length between rear wheels, $w_{v_{\text {WSS }}}$ is the white noise of WSS derived vehicle speed, and $w_{\dot{\psi}_{\text {wss }}}$ is the white noise of WSS derived yaw rate.

The measurement model is generally expressed as follows:

$$
\mathbf{z}=\mathbf{H} \delta \mathbf{x}+\mathbf{v}, \quad \mathbf{v} \sim(0, \mathbf{R})
$$

where $\mathbf{z}$ is the measurement vector, $\mathbf{H}$ is the design matrix, $\delta \mathbf{x}$ is the error state vector, $\mathbf{v}$ is the measurement error, and $\mathbf{R}$ is the variance-covariance matrix of the measurement error.

In this study, the GNSS measurement vector consists of difference between the latitude, the longitude, the north velocity, and the east velocity estimated from DR based on vehicle dynamic sensors and GNSS receiver, as shown below:

$$
\mathbf{z}_{\mathrm{GNSS}}=\left[\begin{array}{c}
\varphi \\
\lambda \\
v_{\mathrm{n}} \\
v_{\mathrm{e}}
\end{array}\right]_{\mathrm{DR}}-\left[\begin{array}{c}
\varphi \\
\lambda \\
v_{\mathrm{n}} \\
v_{\mathrm{e}}
\end{array}\right]_{\mathrm{GNSS}},
$$

where $\mathbf{z}_{\mathrm{GNSS}}$ is the GNSS measurement vector, the subscripts DR and GNSS denote the values computed by DR based on vehicle dynamic sensors and the values calculated from the GNSS receiver, respectively, $\varphi$ and $\lambda$ are the latitude and the longitude, respectively, and $v_{\mathrm{n}}$ and $v_{\mathrm{e}}$ are the north velocity and the east velocity, respectively.

The design matrix and the variance-covariance matrix for GNSS measurements are shown, respectively, as follows:

$$
\begin{aligned}
\mathbf{H}_{\mathrm{GNSS}} & =\left[\begin{array}{lll}
\mathbf{I}_{4 \times 4} & \mathbf{0}_{4 \times 3}
\end{array}\right], \\
\mathbf{R}_{\mathrm{GNSS}} & =\left[\begin{array}{cccc}
\sigma_{\varphi}^{2} & 0 & 0 & 0 \\
0 & \sigma_{\lambda}^{2} & 0 & 0 \\
0 & 0 & \sigma_{v_{\mathrm{n}}}^{2} & 0 \\
0 & 0 & 0 & \sigma_{v_{\mathrm{e}}}^{2}
\end{array}\right],
\end{aligned}
$$

where $\mathbf{H}_{\mathrm{GNSS}}$ is the GNSS design matrix, $\mathbf{R}_{\mathrm{GNSS}}$ is the GNSS variance-covariance matrix, $\mathbf{I}_{4 \times 4}$ is the $4 \times 4$ identity matrix, $\mathbf{0}_{4 \times 3}$ is the $4 \times 3$ zero matrix, and $\sigma_{\varphi}^{2}, \sigma_{\lambda}^{2}, \sigma_{v_{\mathrm{n}}}^{2}$, and $\sigma_{v_{\mathrm{e}}}^{2}$ are the variance of the estimated latitude, the longitude, the north velocity, and the east velocity from GNSS receiver, respectively. 


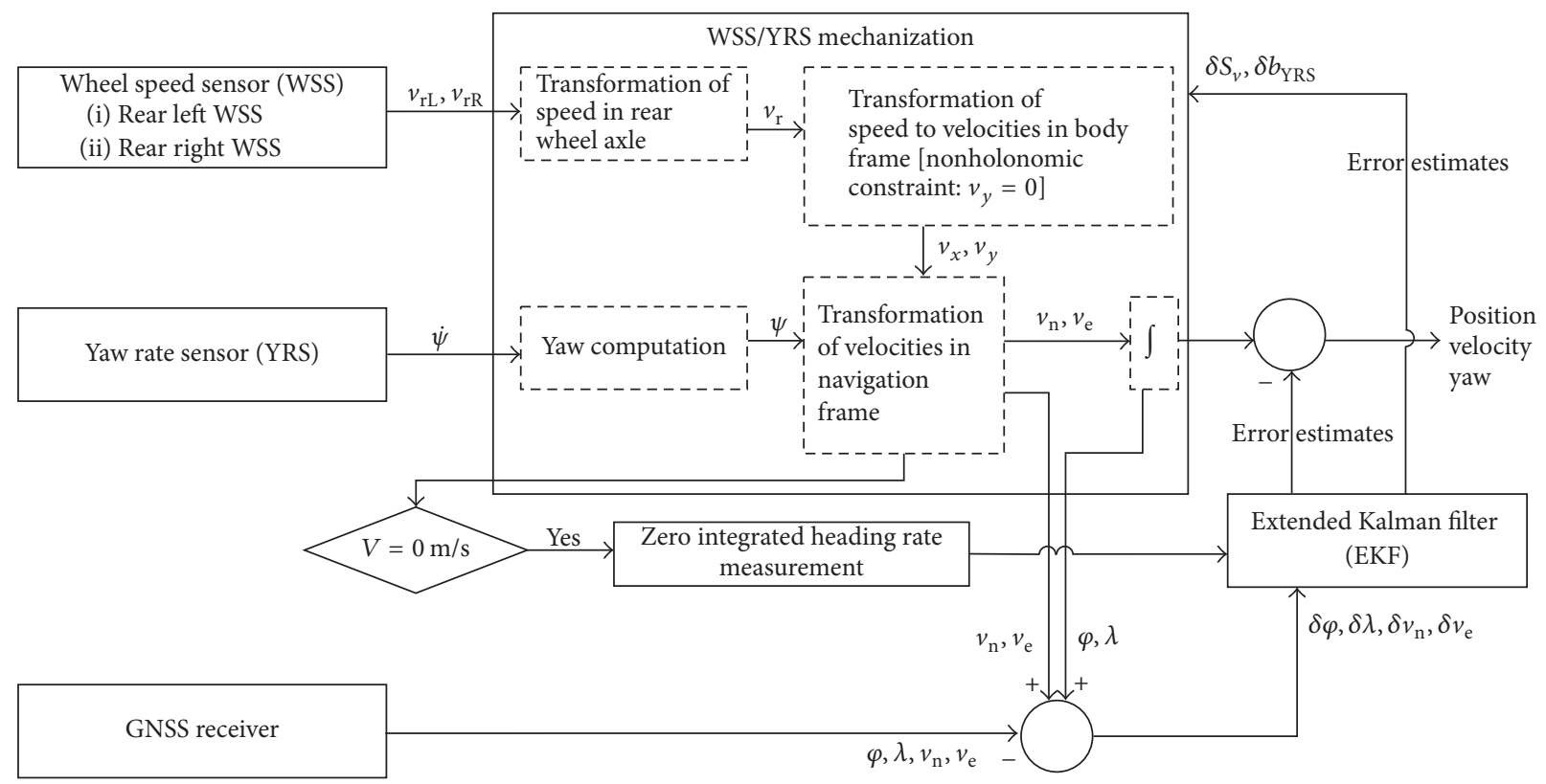

FIGURE 2: Block diagram of GNSS/WSS/YRS integration algorithm.

2.3. GNSS/WSS/YRS Integration Algorithm. Figure 2 shows a block diagram of the GNSS/WSS/YRS integration algorithm. Its structure is similar to that of the GNSS/WSS integration algorithm shown in Figure 1. The major difference between the DR mechanization based on WSS and the DR mechanization based on WSS/YRS is that the yaw rate value is measured from YRS. Therefore, in EKF-based GNSS/WSS/YRS integration, the state vector of the navigation error is the same as that in GNSS/YRS integration, and the state vector of the sensor error is composed of the average of the left and right WSS scale factor and the YRS bias, defined as random constants. The dynamic model for the GNSS/WSS/YRS integration is given in

$$
\begin{aligned}
& \underbrace{\left[\begin{array}{c}
\delta \dot{\varphi} \\
\delta \dot{\lambda} \\
\delta \dot{v}_{\mathrm{n}} \\
\delta \dot{v}_{\mathrm{e}} \\
\delta \dot{\psi} \\
\delta \dot{s}_{v} \\
\delta \dot{b}_{\mathrm{YRS}}
\end{array}\right]=\left[\begin{array}{ccccccc}
0 & 0 & \frac{1}{M+h_{0}} & 0 & 0 & 0 & 0 \\
\frac{v_{\mathrm{e}} \tan \varphi}{\left(N+h_{0}\right) \cos \varphi} & 0 & 0 & \frac{1}{\left(N+h_{0}\right) \cos \varphi} & 0 & 0 & 0 \\
0 & 0 & 0 & 0 & -v_{x} \sin \psi & \cos \psi & 0 \\
0 & 0 & 0 & 0 & v_{x} \cos \psi & \sin \psi & 0 \\
0 & 0 & 0 & 0 & 0 & 0 & 1 \\
0 & 0 & 0 & 0 & 0 & 0 & 0 \\
0 & 0 & 0 & 0 & 0 & 0 & 0
\end{array}\right]}_{=\delta \dot{x}_{\mathrm{WSS} / \mathrm{YRS}}} \underbrace{\left[\begin{array}{ccc}
\delta \mathbf{x}_{\mathrm{WSS} / \mathrm{YRS}} \\
\delta b_{\mathrm{YRS}}
\end{array}\right]}_{=\mathbf{F}_{\mathrm{WSS} / \mathrm{YRS}}} \\
& +\underbrace{\left[\begin{array}{cc}
\frac{\cos \psi}{M+h_{0}} & 0 \\
\frac{\sin \psi}{\left(N+h_{0}\right) \cos \varphi} & 0 \\
0 & 0 \\
0 & 0 \\
0 & 1 \\
0 & 0 \\
0 & 0
\end{array}\right]}_{=\mathbf{G}_{\mathrm{WSS} / \mathrm{YRS}}} \text { } \underbrace{\left[\begin{array}{l}
w_{v_{\mathrm{WSS}}} \\
w_{\dot{\psi}_{\mathrm{YRS}}}
\end{array}\right]}_{=\mathbf{u}_{\mathrm{WSS} / \mathrm{YRS}}},
\end{aligned}
$$




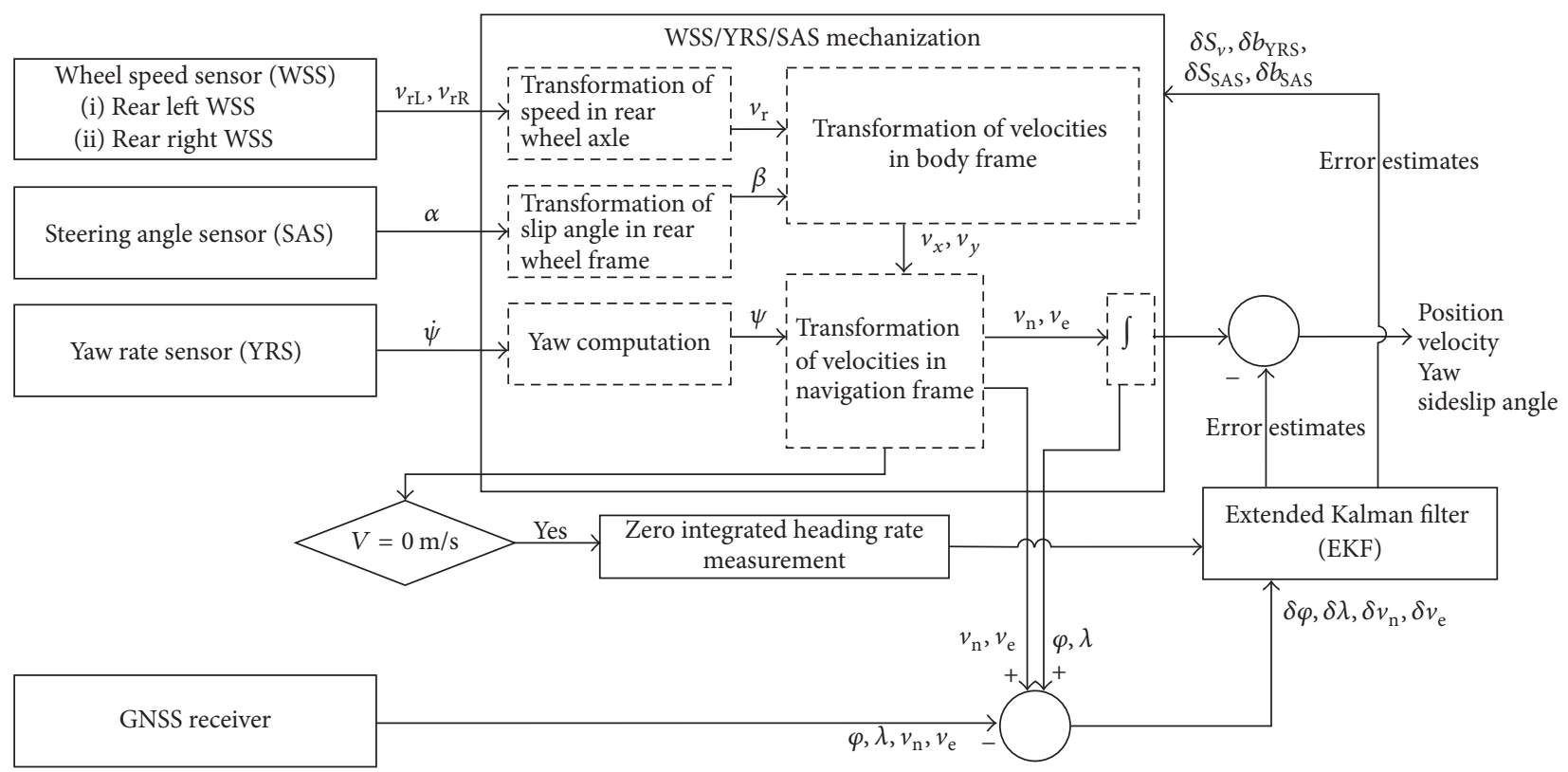

FIGURE 3: Block diagram of GNSS/WSS/YRS/SAS integration algorithm.

where $\mathbf{F}_{\mathrm{WSS} / \mathrm{YRS}}, \delta \mathbf{x}_{\mathrm{WSS} / \mathrm{YRS}}, \mathbf{G}_{\mathrm{WSS} / \mathrm{YRS}}$, and $\mathbf{u}_{\mathrm{WSS} / \mathrm{YRS}}$ are the dynamic matrix, the error state vector, the shaping matrix, and the white noise vector for GNSS/WSS/YRS integration, respectively, $\delta b_{\mathrm{YRS}}$ is the YRS bias, and $w_{\dot{\psi}_{\mathrm{YRS}}}$ is the white noise of YRS.

Comparing the GNSS/WSS integration, the GNSS/WSS/ YRS integration performs not only the GNSS measurement update but also the ZIHR (Zero Integrated Heading Rate) measurement update [3] to prevent the drift error of yaw when the vehicle is stationary. To update the error state vector by using GNSS based position and velocity, the GNSS measurement model is the same as that of GNSS/WSS integration, as shown in (8)-(10). When the vehicle stops, the measured speed by WSS is zero, and thus ZIHR can be applied to fix the yaw. The measurement vector for ZIHR is given by

$$
\mathbf{z}_{\mathrm{ZIHR}}=\psi_{k}-\psi_{k-1},
$$

where $\mathbf{z}_{\mathrm{ZIHR}}$ is the ZIHR measurement vector, $\psi_{k}$ is the estimated yaw at the current time, and $\psi_{k-1}$ is the estimated yaw at the previous time.

The design matrix and the variance-covariance matrix for ZIHR are shown, respectively, as follows:

$$
\begin{aligned}
& \mathbf{H}_{\mathrm{ZIHR}}=\left[\begin{array}{lllllll}
0 & 0 & 0 & 0 & 1 & 0 & 0
\end{array}\right], \\
& \mathbf{R}_{\mathrm{ZIHR}}=\sigma_{\psi}^{2},
\end{aligned}
$$

where $\mathbf{H}_{\mathrm{ZIHR}}$ is the ZIHR design matrix, $\mathbf{R}_{\mathrm{ZIHR}}$ is the ZIHR variance-covariance matrix, and $\sigma_{\psi}^{2}$ is the variance of the estimated yaw.

2.4. GNSS/WSS/YRS/SAS Integration Algorithm. Figure 3 shows a block diagram of the GNSS/WSS/YRS/SAS integration algorithm. The idea of integrating SAS for DR is to compute the side slip angle by using the handle steering angle from SAS and then to employ the estimated side slip angle to set the longitudinal and lateral velocity from the WSS derived vehicle speed. In the case of the integration algorithm in Sections 2.2 and 2.3, a lateral constraint is applied to set the velocity in the body frame using WSS. However, in a land vehicle positioning system, since the lateral nonholonomic constraint is violated by a side slip during either cornering or change in road and tire conditions [14], the positioning accuracy would be degraded. Although the side slip angle is a very complicated phenomenon associated with road and tire conditions as well as high vehicle dynamics including fast driving and sharp turns [15], side slip always occurs during turning due to lateral tire deformation [16]. In order to define the lateral velocity by side slip in the two-dimensional DR based on WSS/YRS/SAS, the side slip angle model is defined in (15) with the assumption of a linear relationship between the side slip angle and the handle steering angle:

$$
\beta=\gamma \cdot \alpha
$$

where $\beta$ is the side slip angle, $\gamma$ is the side slip ratio, and $\alpha$ is the handle steering angle measured by SAS.

Considering the side slip angle, the velocities in the body frame are defined as follows:

$$
\left[\begin{array}{l}
v_{x} \\
v_{y}
\end{array}\right]=\left[\begin{array}{l}
v_{\mathrm{r}} \cos \beta \\
v_{\mathrm{r}} \sin \beta
\end{array}\right] .
$$

In the two-dimensional DR based on WSS/YRS/SAS, the velocities in the navigation frame are given by

$$
\left[\begin{array}{c}
v_{\mathrm{n}} \\
v_{\mathrm{e}}
\end{array}\right]=\left[\begin{array}{cc}
\cos \psi & -\sin \psi \\
\sin \psi & \cos \psi
\end{array}\right]\left[\begin{array}{l}
v_{x} \\
v_{y}
\end{array}\right]=\left[\begin{array}{c}
v_{\mathrm{r}} \cos (\psi+\beta) \\
v_{\mathrm{r}} \sin (\psi+\beta)
\end{array}\right] .
$$


In EKF-based GNSS/WSS/YRS/SAS integration, the state vector of the navigation error is composed of the latitude and longitude error, the north and east velocity error, the yaw error, and the side slip angle error. The state vector of the sensor error consists of the average of the left and right WSS scale factor, the YRS bias, the SAS scale factor, and the SAS bias, defined as random constants. The dynamic model for the GNSS/WSS/YRS/SAS integration is expressed as follows:

$=\underbrace{\left[\begin{array}{c}\delta \dot{\varphi} \\ \delta \dot{\lambda} \\ \delta \dot{v}_{\mathrm{n}} \\ \delta \dot{v}_{\mathrm{e}} \\ \delta \dot{\psi} \\ \delta \dot{\beta} \\ \delta \dot{s}_{v} \\ \delta \dot{b}_{\mathrm{YRS}} \\ \delta \dot{s}_{\mathrm{SAS}}\end{array}\right]}_{=\delta \dot{x}_{\mathrm{WSS} / \mathrm{YRS} / \mathrm{SAS}}}$

\begin{tabular}{|c|c|c|c|c|c|c|c|c|c|c|}
\hline 0 & 0 & $\frac{1}{M+h_{0}}$ & 0 & 0 & 0 & 0 & 0 & 0 & & \\
\hline$\frac{v_{\mathrm{e}} \tan \varphi}{\left(N+h_{0}\right) \cos \varphi}$ & 0 & 0 & $\frac{1}{\left(N+h_{0}\right) \cos \varphi}$ & 0 & 0 & 0 & 0 & 0 & & $\delta \lambda$ \\
\hline 0 & 0 & 0 & 0 & $-v_{x} \sin \psi-v_{y} \cos \psi$ & $-v_{x} \sin \psi-v_{y} \cos \psi$ & $\cos (\psi+\beta)$ & 0 & 0 & & $\delta v_{\mathrm{n}}$ \\
\hline 0 & 0 & 0 & 0 & $v_{x} \cos \psi-v_{y} \sin \psi$ & $v_{x} \cos \psi-v_{y} \sin \psi$ & $\sin (\psi+\beta)$ & 0 & 0 & & $\begin{array}{l}\delta v_{\mathrm{e}} \\
\delta \psi\end{array}$ \\
\hline 0 & 0 & 0 & 0 & 0 & 0 & 0 & 1 & 0 & & $\delta \beta$ \\
\hline 0 & 0 & 0 & 0 & 0 & 0 & 0 & 0 & $\beta$ & L & $\delta s_{v}$ \\
\hline 0 & 0 & 0 & 0 & 0 & 0 & 0 & 0 & 0 & & $\delta b_{\mathrm{YRS}}$ \\
\hline 0 & 0 & 0 & 0 & 0 & 0 & 0 & 0 & 0 & & $\delta s_{\mathrm{SAS}}$ \\
\hline 0 & 0 & 0 & 0 & 0 & 0 & 0 & 0 & 0 & & {$\left[\delta b_{\mathrm{SAS}}\right.$} \\
\hline 0 & 0 & 0 & 0 & 0 & 0 & 0 & 0 & 0 & & $A S$ \\
\hline
\end{tabular}

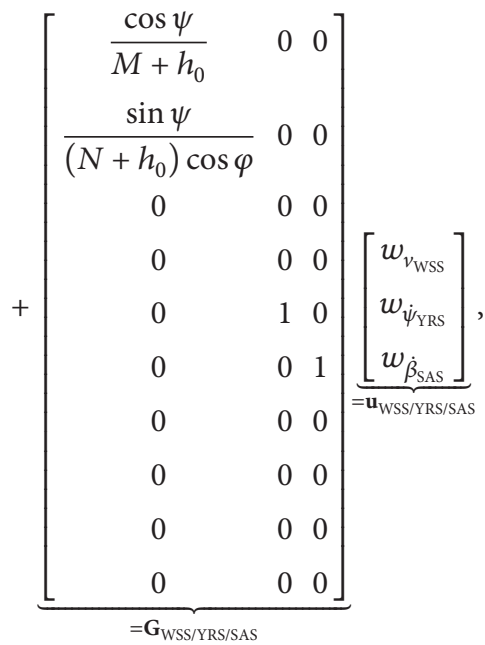




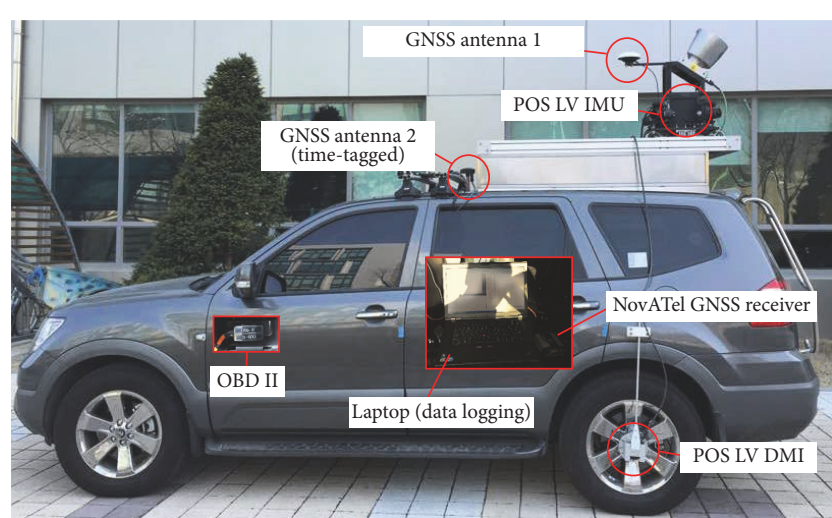

FIGURE 4: Test vehicle.

where $\mathbf{F}_{\text {WSS/YRS/SAS }}, \quad \delta \mathbf{x}_{\text {WSS/YRS/SAS }}, \quad \mathbf{G}_{\mathrm{WSS} / \mathrm{YRS} / \mathrm{SAS}}, \quad$ and $\mathbf{u}_{\text {WSS/YRS/SAS }}$ are the dynamic matrix, the error state vector, the shaping matrix, and the white noise vector for GNSS/WSS/ YRS/SAS integration, respectively, $\delta \beta$ is the side slip angle error, $\delta s_{\text {SAS }}$ is the SAS scale factor, $\delta b_{\text {SAS }}$ is the SAS bias, and $w_{\dot{\beta}_{\mathrm{YRS}}}$ is the white noise of the side slip angle derived SAS.

The measurement vector and the variance-covariance matrix for both the GNSS measurement model and the ZIHR measurement model are the same as that of GNSS/WSS/YRS integration. The design matrices for measurement models in the GNSS/WSS/YRS/SAS integration are expressed as follows:

$$
\begin{aligned}
\mathbf{H}_{\mathrm{GNSS}} & =\left[\begin{array}{ll}
\mathbf{I}_{4 \times 4} & \mathbf{0}_{4 \times 6}
\end{array}\right], \\
\mathbf{H}_{\mathrm{ZIHR}} & =\left[\begin{array}{llllllllllll}
0 & 0 & 0 & 0 & 1 & 0 & 0 & 0 & 0 & 0 & 0 & 0
\end{array}\right] .
\end{aligned}
$$

\section{Performance Evaluation of GNSS/Vehicle Dynamic Sensor Integration Algorithm during GNSS Signal Blockages}

3.1. Test Description. The test vehicle (Figure 4) that is equipped with WSS, SAS, and YRS to operate ADAS is used for this study. The data from the vehicle dynamic sensors are acquired through a CAN (Controller Area Network) bus without installation of additional sensors. The specifications of the vehicle dynamic sensors are described in Table 3. The GNSS receiver used for acquiring the position and the velocity is a NoVATel DL-V3-GENERIC. The positioning method of the GNSS receiver was set to single point positioning using pseudorange and Doppler measurements from GPS and GLONASS. According to the specifications of the GNSS receiver, the precision of position and velocity are $1.5 \mathrm{~m}$ and $0.03 \mathrm{~m} / \mathrm{s}$. To evaluate the performance of the integration algorithms, the reference data were computed in postmission using the inertial, the GNSS, and DMI data obtained from Applanix's POS LV 520. The precision of the horizontal position and yaw for reference data are $0.02 \mathrm{~m}$ and $0.015 \mathrm{deg}$, respectively.

Two trajectories were used in the performance evaluation of the three GNSS/vehicle dynamic sensors integration algorithms during GNSS outages. An experiment for two

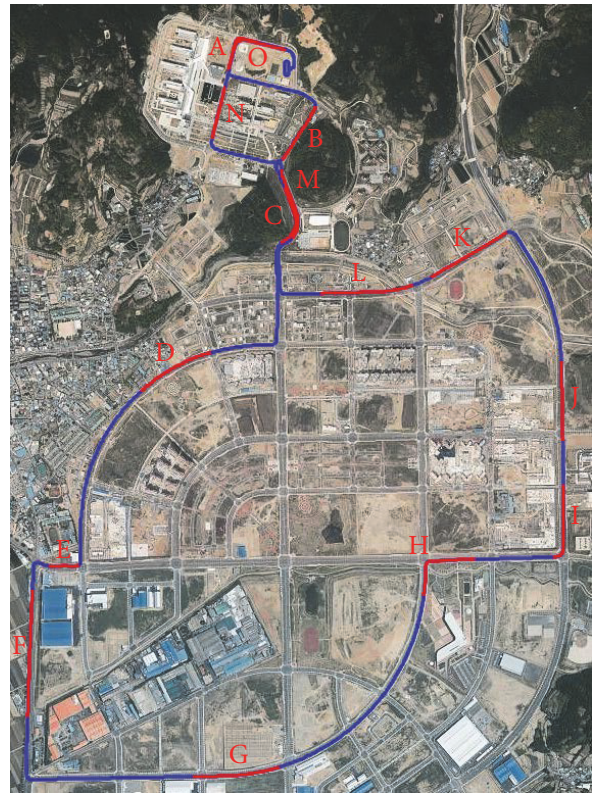

FIGURE 5: The first trajectory (red line: simulated GNSS outage).

trajectories data acquisition was conducted in the Daegu Technopolis area, Korea. These trajectories include various driving circumstances encountered during typical driving on urban roads with frequent stops, acceleration, deceleration, and speed bumps. However, since two trajectories were set in different paths in the same area, the two trajectories data were different in vehicle motion and driving condition. To compare the performance of three GNSS/Vehicle dynamic sensors integration algorithms during GNSS outages, fifteen GNSS outages of 30 seconds were simulated in each trajectory. The simulated GNSS outages covered a wide range of vehicle dynamics such as straight portions, turns, slopes, high speed, slow speeds, and jumping.

3.2. Performance Evaluation in the First Trajectory. Figure 5 shows the reference data of the first trajectory with the simulated GNSS outages shown as a red line overlaid on the trajectory. The traveled distance of the first trajectory is about $11 \mathrm{~km}$ for 32 minutes. Figure 6 shows the velocity of the body frame and the attitude for reference data with the simulated GNSS outages shown as a red line. As can be seen in Figures 5 and 6, the GNSS outages (red line) were chosen by considering different dynamics motions.

First, the drift error of the navigation solution during GNSS outages was analyzed in accordance with the integration strategies. Table 4 compares the average RMS error of the horizontal position at the first and last epoch during GNSS outages by different integration strategies for DR navigation based on the combination of vehicle dynamic sensors. The corresponding results for horizontal velocity and yaw are summarized in Tables 5 and 6. During the GNSS outages, the error of GNSS/WSS integration drifts rapidly. However, the improvement of the horizontal position at the end of the 30 -second GNSS outage is $24 \%$ using the YRS sensor as compared to the GNSS/WSS integration. Furthermore, the 
TABLE 3: Specifications of vehicle dynamic sensors.

\begin{tabular}{lccc}
\hline Vehicle dynamic sensor & Output range & Resolution & Output rate \\
\hline Wheel speed sensor & $0 \sim 511.75 \mathrm{~km} / \mathrm{h}$ & $0.125 \mathrm{~km} / \mathrm{h}$ & $50 \mathrm{~Hz}$ \\
Yaw rate sensor & $-40.95 \sim 40.95 \mathrm{deg} / \mathrm{s}$ & $0.01 \mathrm{deg} / \mathrm{s}$ & $100 \mathrm{~Hz}$ \\
Steering angle sensor & $-3276.8 \sim 3276.6 \mathrm{deg}$ & $0.1 \mathrm{deg}$ & $100 \mathrm{~Hz}$ \\
\hline
\end{tabular}
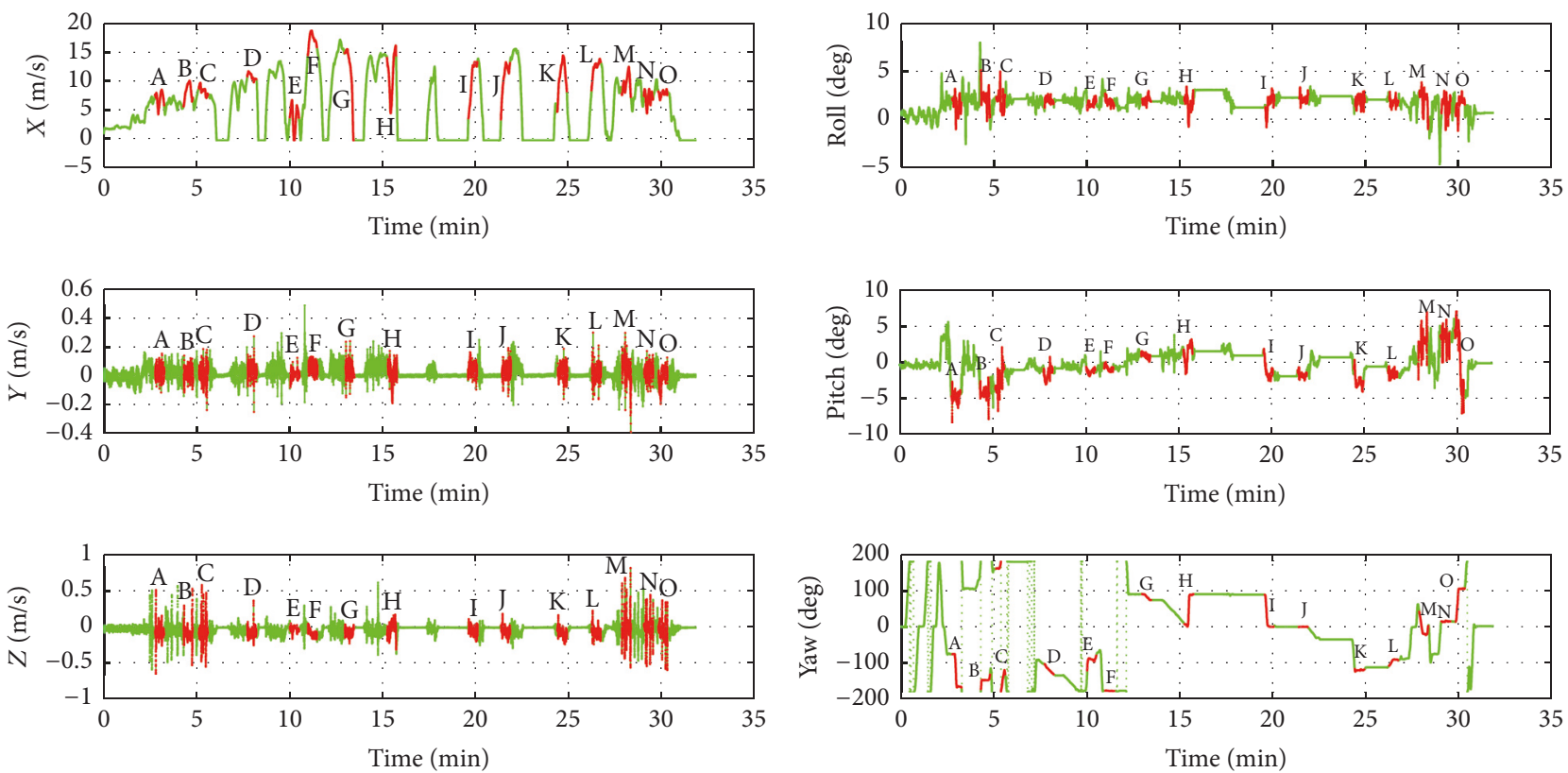

(a) Velocity in body frame

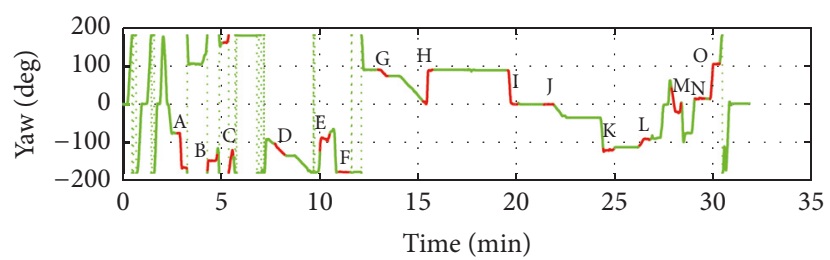

(b) Attitude

FIGURE 6: Velocity and attitude of the first trajectory (red line: simulated GNSS outage).

TABLE 4: Average RMS error of horizontal position during GNSS outages for first trajectory.

\begin{tabular}{lccr}
\hline \multirow{2}{*}{ Integration strategies } & \multicolumn{2}{c}{ Average of RMS error of horizontal position during GNSS outages [m] } \\
& First epoch & Last epoch & Difference (last - first) \\
\hline GNSS/WSS & 2.03 & 9.16 & 7.13 \\
GNSS/WSS/YRS & 1.77 & 7.18 & 5.41 \\
GNSS/WSS/YRS/SAS & 1.75 & 6.09 & 4.34 \\
\hline
\end{tabular}

TABLE 5: Average RMS error of horizontal velocity during GNSS outages for first trajectory.

\begin{tabular}{|c|c|c|c|}
\hline \multirow{2}{*}{ Integration strategies } & \multicolumn{3}{|c|}{ Average of RMS error of horizontal velocity during GNSS outages [m/s] } \\
\hline & First epoch & Last epoch & Difference (last - first) \\
\hline GNSS/WSS & 0.25 & 0.43 & 0.18 \\
\hline GNSS/WSS/YRS & 0.22 & 0.28 & 0.06 \\
\hline GNSS/WSS/YRS/SAS & 0.22 & 0.26 & 0.04 \\
\hline
\end{tabular}

TABLE 6: Average RMS error of yaw during GNSS outages for first trajectory.

\begin{tabular}{lccr}
\hline Integration strategies & & Average of RMS error of yaw during GNSS outages [deg] \\
& First epoch & Last epoch & Difference (last - first) \\
\hline GNSS/WSS & 1.07 & 2.61 & 1.54 \\
GNSS/WSS/YRS & 0.74 & 1.74 & 1.00 \\
GNSS/WSS/YRS/SAS & 0.60 & 1.41 & 0.81 \\
\hline
\end{tabular}


TABLE 7: Cumulative relative frequency table of maximum horizontal position error for GNSS outages of first trajectory.

\begin{tabular}{lcr}
\hline Integration strategies & \multicolumn{2}{c}{ Maximum horizontal position error } \\
\hline GNSS/WSS & $27 \%$ (4 out of total 15 outages) & $87 \%$ (13 out of total 15 outages) \\
GNSS/WSS/YRS & $33 \%$ (5 out of total 15 outages) & $93 \%$ (14 out of total 15 outages) \\
GNSS/WSS/YRS/SAS & $53 \%$ (8 out of total 15 outages) & $93 \%$ (14 out of total 15 outages) \\
\hline
\end{tabular}

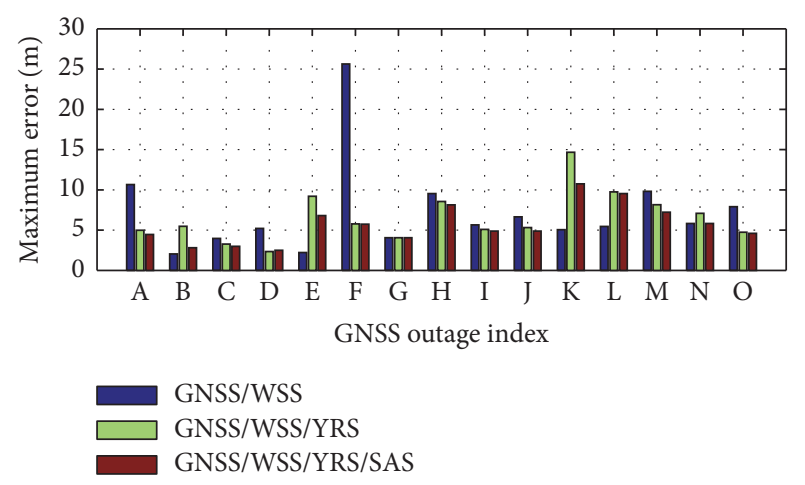

FIGURE 7: Maximum horizontal position error for GNSS outages of first trajectory.

average RMS error of horizontal velocity at the end of the 30 -second GNSS outage is improved by about $67 \%$. This is due to the dependency of the limitation of WSS accuracy for yaw computation on factors such as wheel slipping, skidding, and slope. Comparing GNSS/WSS/YRS integration with GNSS/WSS/YRS/SAS integration, application of SAS based side slip angle in DR improves the positioning performance by about $20 \%$ during GNSS outages. Also, the average RMS error of horizontal velocity and yaw is reduced by $66 \%$ and $19 \%$, respectively, from $0.06 \mathrm{~m} / \mathrm{s}$ and 1.00 degrees for GNSS/WSS/YRS integration to $0.04 \mathrm{~m} / \mathrm{s}$ and 0.81 degrees for GNSS/WSS/YRS/SAS integration. This indicates that the computation of the SAS based side slip angle could be employed to alleviate the effect of violating the lateral nonholonomic constraints.

The maximum horizontal position error values during the fifteen outages are presented in Figure 7. The cumulative relative frequency based on the maximum horizontal position error of $5 \mathrm{~m}$ and $10 \mathrm{~m}$ is summarized in Table 7. As expected, the integration strategy with all the vehicle dynamic sensors together (GPS/WSS/YRS/SAS) shows the overall best performance. Despite this, the maximum horizontal error calculated by the three GNSS/vehicle dynamic sensors integration algorithms was generally smaller than $10 \mathrm{~m}$ during the GNSS outage for 30 seconds. However, in the case of GNSS/WSS integration, the largest horizontal position error was observed for GNSS outage F. In this outage, the vehicle is traveling along a straight path, and the vehicle speed is greater than the other GNSS outages, as shown in both Figure 5 and Figure 6. Figure 8 shows the details of the horizontal position error, the longitudinal velocity error, and the yaw error during GNSS outages $F$. The longitudinal velocity error of three GNSS/Vehicle dynamic
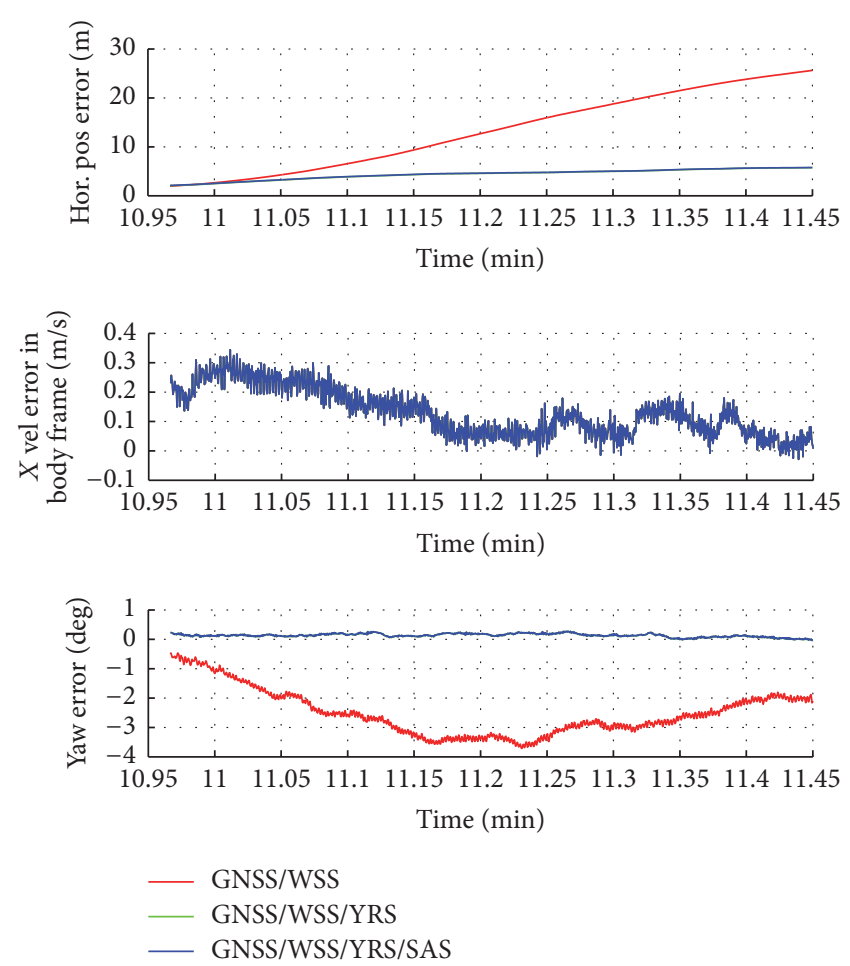

FIGURE 8: Details of the horizontal position error, the longitudinal velocity error, and the yaw error during GNSS outages F.

sensors integration algorithms were almost the same in the GNSS outages F. Despite this, the horizontal position error of the GNSS/WSS integration was higher than the other integrations due to the yaw error. As mentioned in Section 2, the major difference between GNSS/WSS integration and GNSS/WSS/YRS integration in DR mechanization is that the yaw computation in GNSS/WSS uses the calculated yaw rate based on the difference between the rear left wheel speed and the rear right wheel speed. If the vehicle is traveling along a straight path on flat road, the performance of GNSS/WSS integration is similar to the performance of GNSS/WSS/YRS integration because the yaw rate calculated by the rear wheels is zero in the ideal situation. However, since the wheel slipping and skidding in the left and right wheels could occurred differently due to the influence of either uneven road surface or vehicle dynamic force, the yaw rate calculated by the rear wheels is not zero in the real situation. This means that the accuracy of the computed yaw angle by using WSS could be significantly degraded. For this result, it is considered that WSS based DR has limited application to car positioning. 
TABLE 8: Average RMS error of horizontal position during GNSS outages for second trajectory.

\begin{tabular}{|c|c|c|c|}
\hline \multirow{2}{*}{ Integration strategies } & \multicolumn{3}{|c|}{ Average of RMS error of horizontal position during GNSS outages [m] } \\
\hline & First epoch & Last epoch & Difference (last - first) \\
\hline GNSS/WSS & 1.91 & 15.54 & 13.62 \\
\hline GNSS/WSS/YRS & 1.65 & 5.86 & 4.22 \\
\hline GNSS/WSS/YRS/SAS & 1.64 & 5.57 & 3.93 \\
\hline
\end{tabular}

TABLE 9: Average RMS error of horizontal velocity during GNSS outages for second trajectory.

\begin{tabular}{|c|c|c|c|}
\hline \multirow{2}{*}{ Integration strategies } & \multicolumn{3}{|c|}{ Average of RMS error of horizontal velocity during GNSS outages $[\mathrm{m} / \mathrm{s}]$} \\
\hline & First epoch & Last epoch & Difference (last - first) \\
\hline GNSS/WSS & 0.16 & 0.97 & 0.81 \\
\hline GNSS/WSS/YRS & 0.12 & 0.32 & 0.20 \\
\hline GNSS/WSS/YRS/SAS & 0.12 & 0.28 & 0.17 \\
\hline
\end{tabular}

TABLE 10: Average RMS error of yaw during GNSS outages for second trajectory.

\begin{tabular}{lccc}
\hline Integration strategies & & Average of RMS error of yaw during GNSS outages [deg] \\
& First epoch & Last epoch & 5.75 \\
GNSS/WSS & 0.52 & 2.88 & 5.23 \\
GNSS/WSS/YRS & 0.36 & 2.80 & 2.52 \\
GNSS/WSS/YRS/SAS & 0.34 & 2.46 \\
\hline
\end{tabular}

3.3. Performance Evaluation in the Second Trajectory. The test data for the second trajectory were acquired by driving a different path of $15.6 \mathrm{~km}$ for $39 \mathrm{~min}$. The GNSS outages were selected by the same criteria. Figure 9 shows the reference data of the second trajectory with the fifteen simulated GNSS outages. Both the velocity in the body frame and the attitude for reference data are shown in Figure 10. Compared with the first trajectory, the driving direction and the velocity for the second trajectory were changed more frequently.

Table 8 presents the average RMS error of the horizontal position at the first and last epoch during GNSS outages with respect to the integration strategies. Tables 9 and 10 summarize the average RMS error of the horizontal velocity and yaw at the first and last epoch during GNSS outages, respectively. The average RMS of the horizontal position of GNSS/WSS integration is significantly increased in comparison with the results of the first trajectory, as shown in Table 4. Despite this, the horizontal position RMS error of the GNSS/WSS integration of $13.62 \mathrm{~m}$ at the end of the 30-second GNSS outage was reduced to $4.22 \mathrm{~m}$ by using the WSS plus YRS, constituting a 69\% improvement. The horizontal velocity drift error can be reduced from $0.81 \mathrm{~m} / \mathrm{s}$ for GNSS/WSS integration to $0.20 \mathrm{~m} / \mathrm{s}$ through the integration with YRS, and the yaw drift error is reduced from 5.23 degrees to 2.52 degrees. The horizontal velocity percentage improvement is $75 \%$ and the yaw percentage improvement is $52 \%$. Therefore, using the yaw rate measured by YRS instead of that computed by WSS greatly enhances the DR accuracy during GNSS outages. The average of RMS horizontal position errors of the GNSS/WSS/YRS/SAS integration at the end of the 30-second GNSS outage is improved by about 7\% compared to the GNSS/WSS/YRS

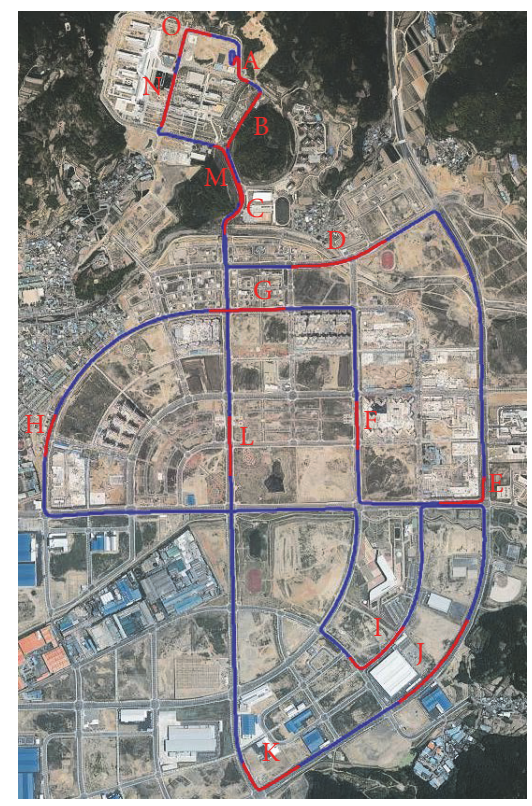

Figure 9: The second trajectory (red line: simulated GNSS outage).

solution. Furthermore, for the GNSS/WSS/YRS integration, the enhancement at the end of the 30-second GNSS outages is $15 \%$ for the average RMS error of horizontal velocity and $2 \%$ for the average RMS error of the yaw case as compared to the GNSS/WSS/YRS solution. Although the improvement gained from additional integration of the SAS is slight, using the SAS based side slip angle for DR can reduce the drift error. 
TABLE 11: Cumulative relative frequency table of maximum horizontal position error for GNSS outages of second trajectory.

\begin{tabular}{|c|c|c|}
\hline \multirow{2}{*}{ Integration strategies } & \multicolumn{2}{|c|}{ Maximum horizontal position error } \\
\hline & $\leq 5 \mathrm{~m}$ & $\leq 10 \mathrm{~m}$ \\
\hline GNSS/WSS & $27 \%$ (4 out of total 15 outages) & $60 \%$ ( 9 out of total 15 outages) \\
\hline GNSS/WSS/YRS & $53 \%$ ( 8 out of total 15 outages) & $93 \%$ (14 out of total 15 outages) \\
\hline GNSS/WSS/YRS/SAS & $67 \%$ (10 out of total 15 outages) & $93 \%$ (14 out of total 15 outages) \\
\hline
\end{tabular}
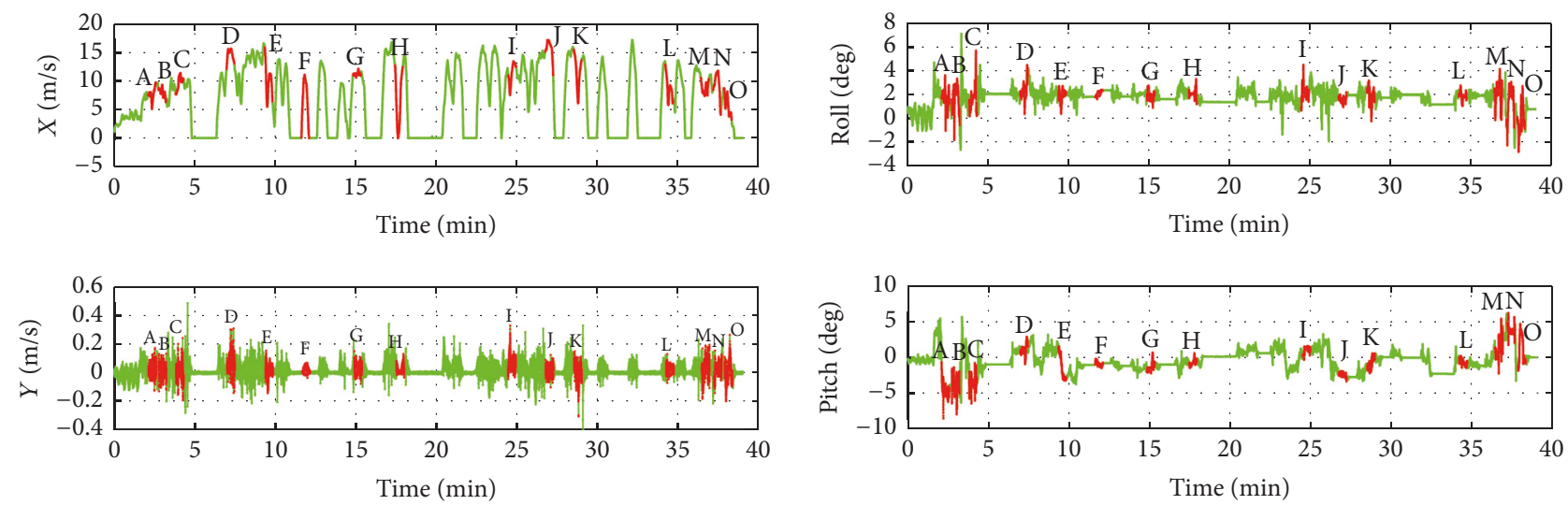

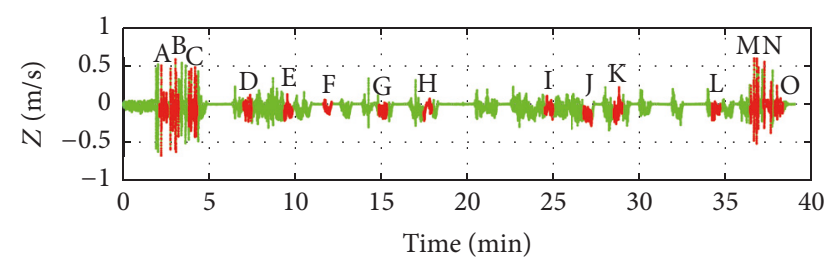

(a) Velocity in body frame

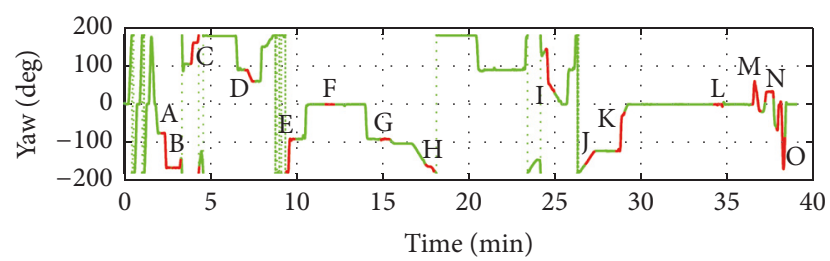

(b) Attitude

FIGURE 10: Velocity and attitude of the first trajectory (red line: simulated GNSS outage).
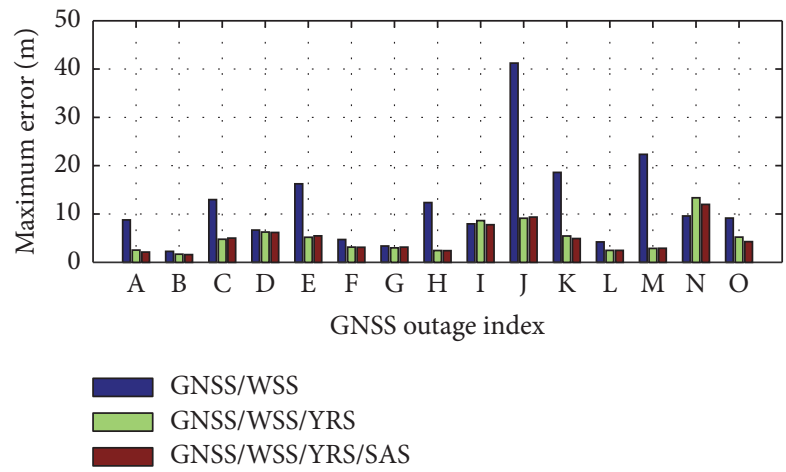

FIGURE 11: Maximum horizontal position error for GNSS outages of second trajectory.

Figure 11 shows the maximum horizontal position error during the fifteen outages. The cumulative relative frequency based on the maximum horizontal position error of $5 \mathrm{~m}$ and $10 \mathrm{~m}$ is summarized in Table 11. The maximum horizontal position error for GNSS/WSS integration is smaller than $10 \mathrm{~m}$ in nine out of the total 15 GNSS outages (60\%), which is worse than the result of the first trajectory. It can be seen that the accuracy of the computed yaw rate using WSS is easily degraded by typical conditions encountered by a land vehicle, including uneven road, speed bumps, high speed, and sudden acceleration and deceleration. As seen in Figure 10, for the velocity and attitude of GNSS outages A, C, E, H, J, K, and $\mathrm{L}$, where the maximum horizontal error of GNSS/WSS integration is higher than the other integrations, the accuracy of the computed yaw rate using WSS is easily degraded by typical driving conditions encountered by a land vehicle, including uneven road, speed bumps, high speed, and sudden acceleration, and deceleration. Therefore, it is estimated that the GNSS/WSS integration would provide an unstable navigation solution during GNSS outages. The maximum horizontal position error for GNSS/WSS/YRS integration as well as GNSS/WSS/YRS/SAS integration is smaller than $10 \mathrm{~m}$ in fourteen out of the total 15 GNSS outages (93\%), which is equal to that of the first trajectory. Also, GNSS/WSS/YRS/SAS integration achieves the overall best performance.

\section{Conclusions}

This study presents the performance evaluation of a land vehicle positioning system encompassing GNSS combined with a two-dimensional DR based on vehicle dynamic sensors. To develop GNSS/vehicle dynamic sensor based positioning algorithms, vehicle dynamic sensors used WSS, YRS, and SAS, which were already installed in the test vehicle. 
Three two-dimensional DR mechanisms were designed to ensure that the vehicle dynamic sensors' information is not duplicated. The GNSS/vehicle dynamic sensors integrations were implemented by EKF through a loosely coupled mode. The GNSS measurement are the position and the velocity calculated using the C/A code and the Doppler measurements from GPS and GLONASS at a GNSS receiver. The developed algorithms were tested on two trajectories acquired in various driving circumstances. A performance evaluation was conducted in fifteen simulated GNSS outages during 30 seconds for each trajectory. The results indicate that the integration algorithm with all the vehicle dynamic sensors together (GPS/WSS/YRS/SAS) provided the best performance. With respect to two trajectories, the maximum horizontal position error of both GNSS/WSS/YRS and GNSS/WSS/YRS/SAS integration was smaller than $10 \mathrm{~m}$ in 28 out of the total 30 GNSS outages. And the maximum horizontal positon error of GNSS/WSS integration was smaller than $10 \mathrm{~m}$ in 22 out of the total 30 GNSS outages. It is estimated that the GNSS/WSS integration would provide an unstable navigation solution during GNSS outages in comparison to both GNSS/WSS/YRS and GNSS/WSS/YRS/SAS integration since the accuracy of the computed yaw angle by using WSS could be significantly degraded due to frequent wheel slipping and skidding. Therefore, the proposed GNSS/vehicle dynamics sensor integrations excluding GNSS/WSS integration could be applied to an automotive navigation system with meterlevel accuracy to overcome the limitations of the GNSS based positioning technique. Indeed, these algorithms might provide alternative solutions to the use of a low-cost MEMSbased IMU.

\section{Conflicts of Interest}

The authors declare that they have no conflicts of interest.

\section{Acknowledgments}

This work was supported by the DGIST R\&D Program of the Ministry of Science, ICT \& Technology of Korea (17-NT01) for Joong-hee Han and Chi-ho Park. Also, this work was supported by the 2016 Research Fund of the University of Seoul for Jay Hyoun Kwon.

\section{References}

[1] M. Spangenberg, Safe navigation for vehicles [Ph.D. thesis], Toulouse University, Toulouse, France, 2009.

[2] U. Iqbal, Multi-sensor data fusion for vehicular navigation applications [Ph.D. thesis], Queen's University, Kingston, Canada, 2012.

[3] E. Shin, Estimation techniques for low-cost inertial navigation [Ph.D. thesis], The University of Calgary, Calgary, Canada, 2005.

[4] S. Godha and M. E. Cannon, "GPS/MEMS INS integrated system for navigation in urban areas," GPS Solutions, vol. 11, no. 3, pp. 193-203, 2007.

[5] A. G. Quinchia, G. Falco, E. Falletti, F. Dovis, and C. Ferrer, "A comparison between different error modeling of MEMS applied to GPS/INS integrated systems," Sensors, vol. 13, no. 8, pp. 95499588, 2013.

[6] K. Jo, M. Lee, and M. Sunwoo, "Road slope aided vehicle position estimation system based on sensor fusion of GPS and automotive onboard sensors," IEEE Transactions on Intelligent Transportation Systems, vol. 17, no. 1, pp. 250-263, 2016.

[7] D. Barbosa, A. Lopes, and R. E. Araújo, "Sensor fusion algorithm based on Extended Kalman Filter for estimation of ground vehicle dynamics," in Proceedings of the 42nd Conference of the Industrial Electronics Society (IECON '16), pp. 1049-1054, Florence, Italy, October 2016.

[8] M. Darms and H. Winner, "A modular system architecture for sensor data processing of ADAS applications," in Proceedings of the 2005 IEEE Intelligent Vehicles Symposium, pp. 729-734, Las Vegas, Nev, USA, June 2005.

[9] P. Bonnifait, P. Bouron, D. Meizel, and P. Crubillé, "Dynamic localization of car-like vehicles using data fusion of redundant ABS sensors," The Journal of Navigation, vol. 56, no. 3, pp. 429441, 2003.

[10] J. Georgy, A. Noureldin, M. J. Korenberg, and M. M. Bayoumi, "Modeling the stochastic drift of a MEMS-based gyroscope in gyro/odometer/GPS integrated navigation," IEEE Transactions on Intelligent Transportation Systems, vol. 11, no. 4, pp. 856-872, 2010.

[11] S. Rezaei and R. Sengupta, "Kalman filter-based integration of DGPS and vehicle sensors for localization," IEEE Transactions on Control Systems Technology, vol. 15, no. 6, pp. 1080-1088, 2007.

[12] K. Jo, K. Chu, and M. Sunwoo, "Interacting multiple model filter-based sensor fusion of GPS with in-vehicle sensors for real-time vehicle positioning," IEEE Transactions on Intelligent Transportation Systems, vol. 13, no. 1, pp. 329-343, 2012.

[13] P. D. Grove, Principles of GNSS, Inertial, and Multisensor Integrated Navigation System, Artech House, London, UK, 2013.

[14] G. Dissanayake, S. Sukkarieh, E. Nebot, and H. Durrant-Whyte, "The aiding of a low-cost strapdown inertial measurement unit using vehicle model constraints for land vehicle applications," IEEE Transactions on Robotics and Automation, vol. 17, no. 5, pp. 731-747, 2001.

[15] J. Gao, Development of a precise GPS/INS/on-board vehicle sensors integrated vehicular positioning system [Ph.D. thesis], The University of Calgary, Calgary, Canada, 2007.

[16] M. Doumiati, A. Victorino, A. Charara, and D. Lechner, "A method to estimate the lateral tire force and the sideslip angle of a vehicle: experimental validation," in Proceedings of the American Control Conference (ACC '10), pp. 6936-6942, Baltimore, Md, USA, July 2010. 


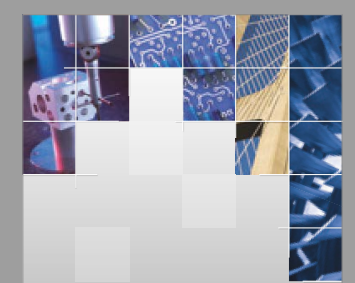

\section{Enfincering}
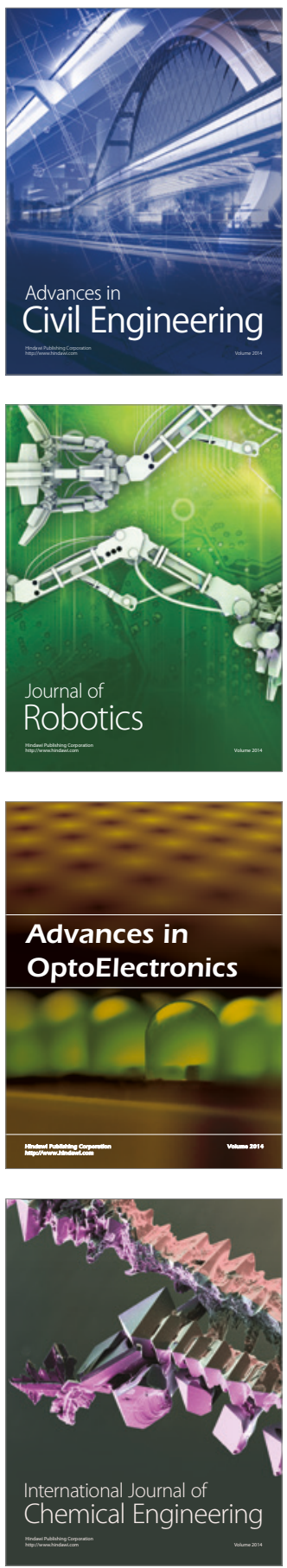

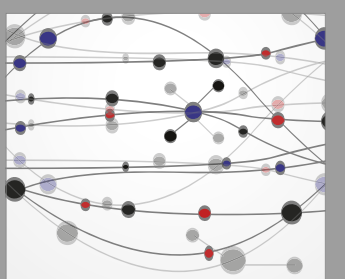

The Scientific World Journal

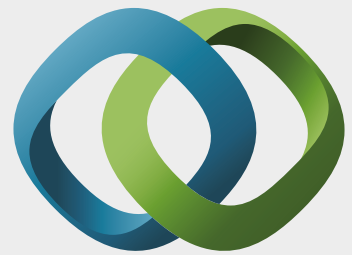

\section{Hindawi}

Submit your manuscripts at

https://www.hindawi.com
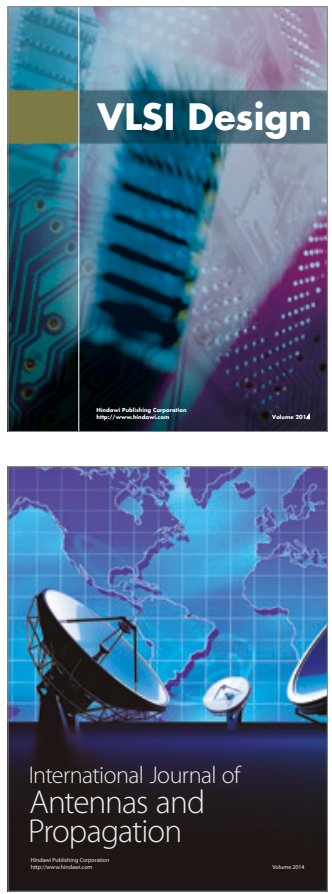

\section{Rotating}

Machinery
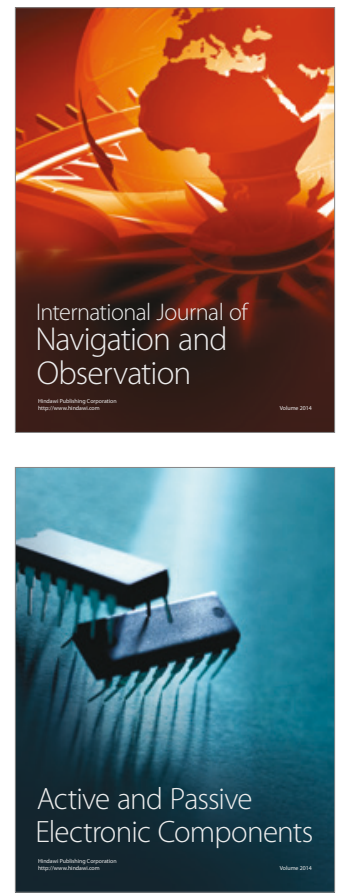
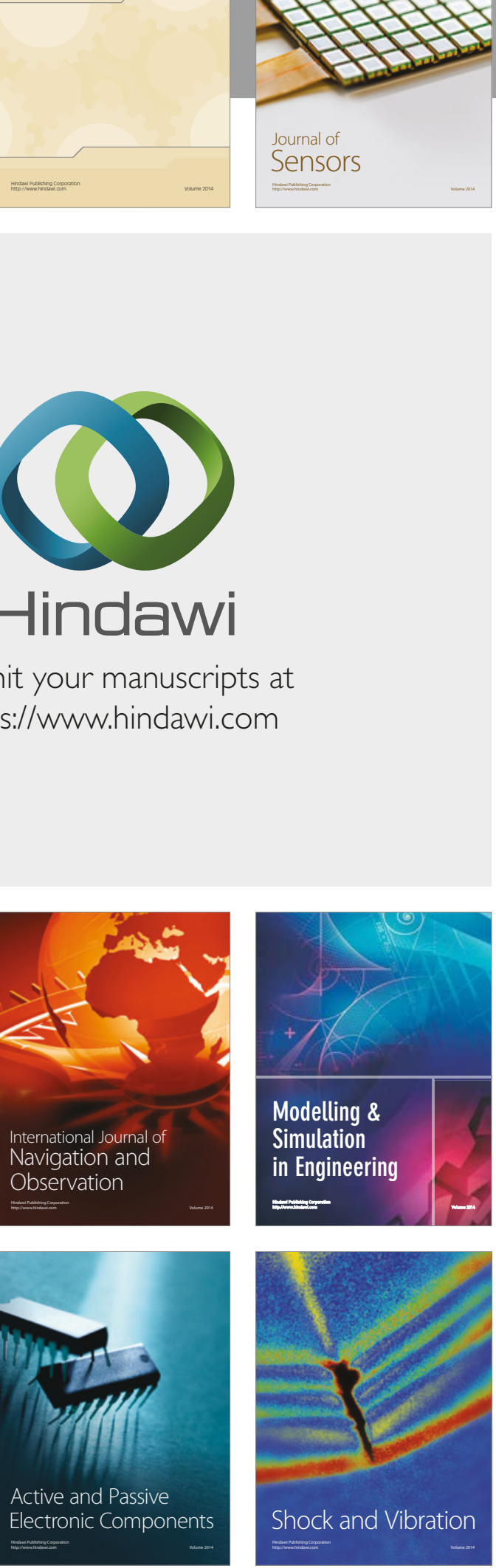
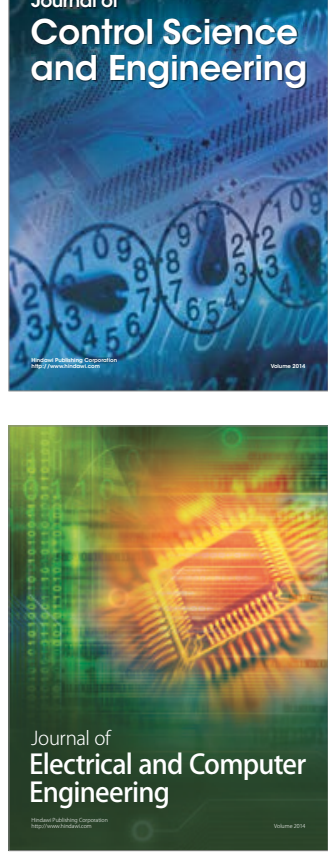

Distributed

Journal of

Control Science

and Engineering
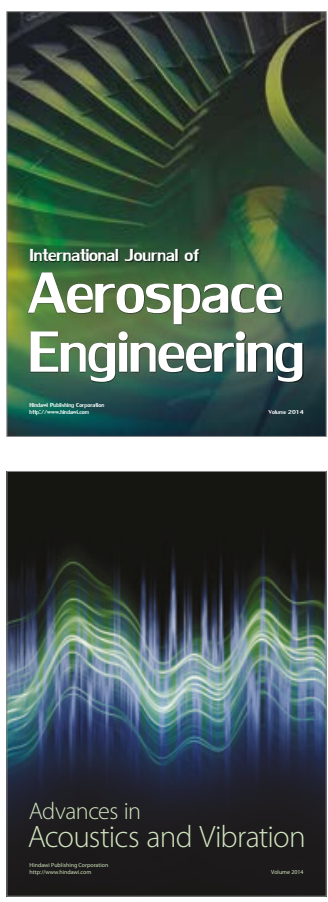

Sensor Networks 